\title{
Quantitative Analysis of Surface Irregularities Resulting from Flow-induced Separation of Highly Filled Compounds
}

\author{
Berenika Hausnerová and Vladimír Pata ${ }^{\mathrm{b}}$ \\ ${ }^{a}$ Centre of Polymer Systems, Department of Production Engineering, Tomas Bata University in Zlin, \\ nám. T.G. Masaryka 5555, 76001 Zlín, Czech Republic \\ ${ }^{b}$ Department of Production Engineering, Tomas Bata University in Zlin, \\ nám. T.G. Masaryka 5555, 76001 Zlín, Czech Republic
}

\begin{abstract}
During shear flow of highly filled (about 60 vol.\% powder particles) polymer melts the occurring separation of particular material components has a detrimental effect on the surface quality of the resulting parts. Currently, there is a lack of reliable simulation approaches available to optimize the processing of such rheologically complex materials as well as tools describing separation in a quantitative way. The aim of the current research is to provide an approach to analyze this phenomenon quantitatively, and compare tendency to separation as a function of material composition and/or processing parameters. In this paper, surface quality of the resulting parts is determined with a contactless scanner with a First Interface Detection testing mode in order to obtain fractal dimensions of reject/fine surface structure of the final products.
\end{abstract}

Keywords: composite, particles, injection molding, surface, contactless scanner, fractal dimension

PACS: 81

\section{INTRODUCTION}

Separation of particular material components during high-shear technologies as injection molding is often the factor influencing quality of the final highly filled products. This is even more severe for parts produced via powder injection molding (PIM), where a polymeric component is thermally or chemically extracted after injection molding, and remaining porous structure is sintered so as to obtain purely metal or ceramic item. A considerable portion of final parts does not meet the quality requirements due to the separation of feedstock components - polymer component(s) and powder during molding.

According to Thornagel [1] local shear rate gradients are the driving forces initiating phase separation. If a feedstock has a good adhesion to the flow channel, such gradients occur in a close proximity of the wall, while low shear rate plateau is concentrated in the middle of the flow domain. At high shear regions powder particles start to rotate to leave away, resulting in inhomogeneous structure composed of polymer-rich and powder-rich areas.

Regardless an effort spent in the last decade to describe and simulate the separation, there is still no sophisticated solution of such quality-determining factor. Since the separation occurs at injection molding stage, the key to its reduction/elimination lays in the flow behavior during high shear deformation in the flow channels and mold. The testing molds employed up to day only partly fulfill the purpose. Thus, a new design of the testing mold [2] has been developed at the Polymer Centre TBU in Zlín (in cooperation with Fraunhofer IFAM, Bremen) and mold constructed in order to intercept critical geometrical issues like inner and outer corners, radical thickness changes, weldlines and very thin parts.

Further, recently we have shown that scanning electron microscopy (SEM) of particular cross sections derived from this testing mold combined with energy dispersive X-ray (EDX) analysis of the distribution of elements typical for powder and polymer(s) might provide a quantitative evaluation of separation [3]. In comparison to an approach introduced by Jenni et al. [4], where differential scanning calorimetry (DSC) was selected to quantify the local powder concentration, SEM-EDX analysis offers quantitatively significant results. A DSC approach also cannot be

6th International Conference on Times of Polymers (TOP) and Composites

AIP Conf. Proc. 1459, 145-147 (2012); doi: 10.1063/1.4738425

(C) 2012 American Institute of Physics 978-0-7354-1061-9/\$30.00 
used for the cases, where polymer component is a mixture of various polymers with similar transition temperatures as often with PIM compounds, where a step-by-step extraction prior sintering is required.

In this paper the attention is paid to the quantitative analysis of surface structures resulting from the separation.

\section{RESULTS AND DISCUSSION}

The methods to observe results of separation on final parts (after polymer extraction and sintering) employed so far, relay largely on electron microscopy. In order to obtain a quantitative analysis we propose a contactless 3D Chromatic Length Aberration (CLA) scanner (Talysurf 300, Taylor and Hobson, UK) equipped with Talymap ver.5.0.2 software.

Tested surfaces (Figure 1) were subjected to a height measurement over a rectangular area $(500 \times 500) \mu \mathrm{m}$. The data supplied is of the form $z=\mathrm{f}(x, y)$, where $z$ is the height of the profile, $x$ stands for the position over the scanning direction, and $y$ corresponds to the number of traces. First Interface Detection (FID) was selected as a measurement mode. The software takes into account the height of the first interference (i.e. the upper border of the transparent interference represented by the first peak in the spectrum), Figure 2.

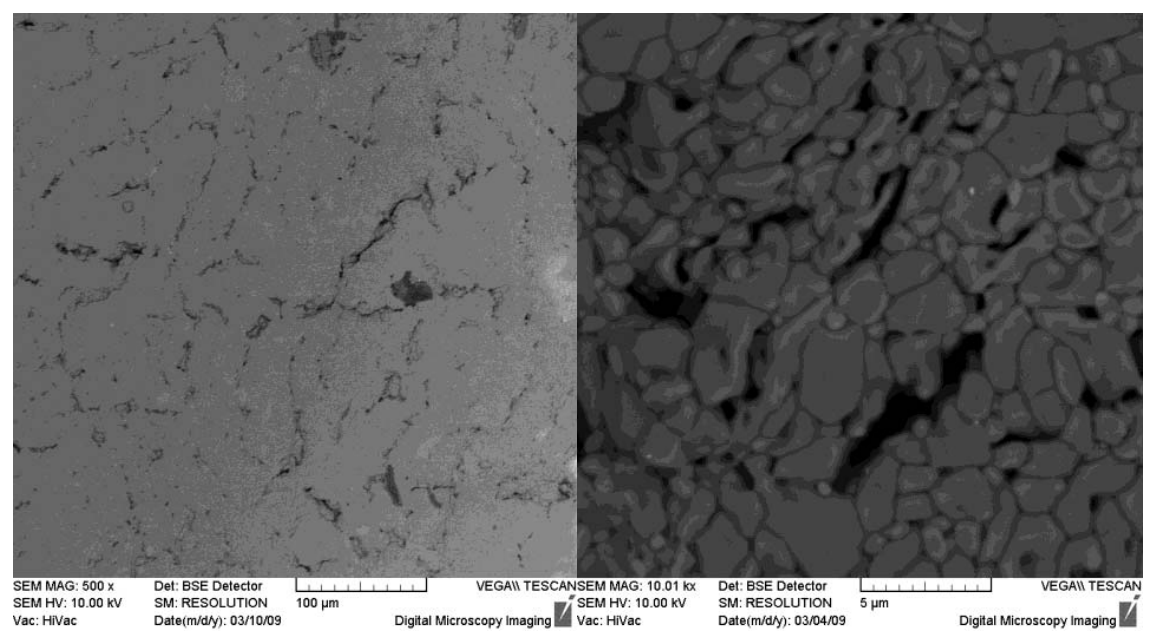

FIGURE 1. SEM micrographs of the final structure of sintered highly compressive superground aluminum oxide (MARTOXID ${ }^{\circledR}$ MR70, Albemarle Corporation, USA); on the right - the detail of voids resulting from the separated polymer areas.
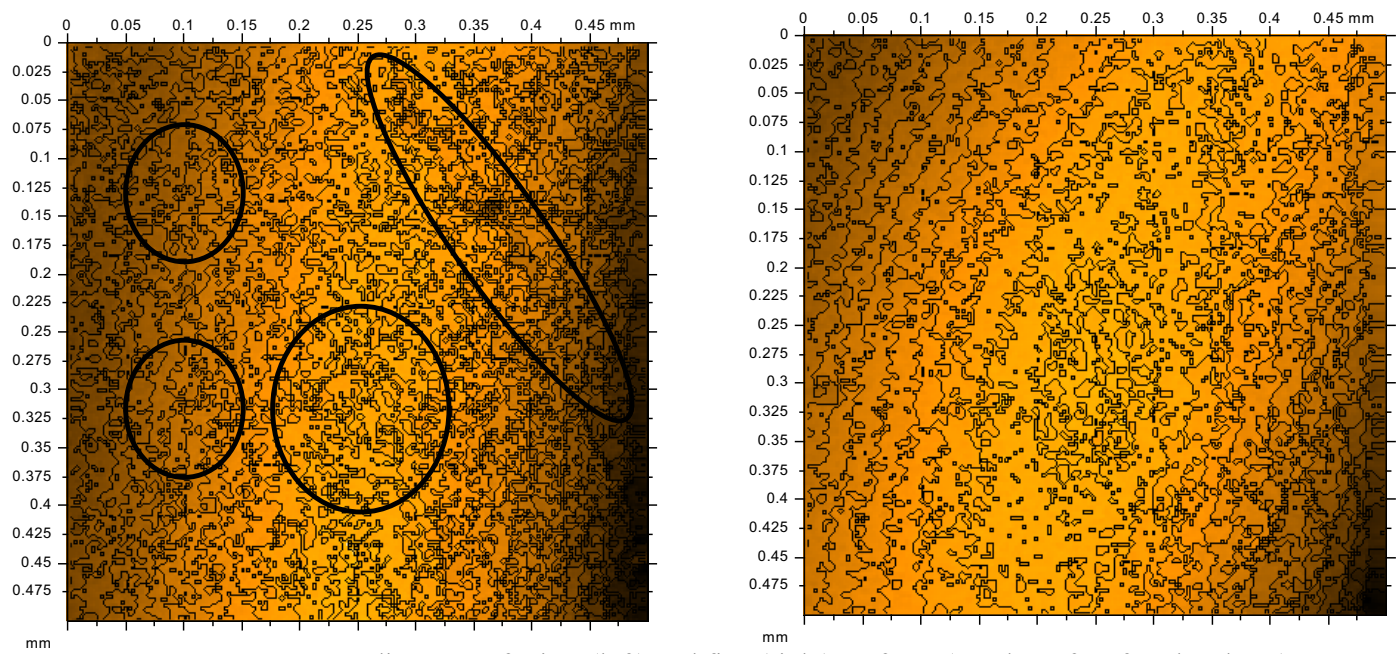

FIGURE 2. Contour diagrams of reject (left) and fine (right) surfaces (number of surface levels 20). 
Figure 2 shows the highland maps of reject and fine surfaces with highlighted surface defects' areas on the reject part. A method of including boxes was selected to determine the fractal dimensions of the surfaces. The method is based on enclosing each section of the profile by a box with defined width and calculation of the area of all boxes enclosing the profile. From the results (Figure 3) the quantitative description of the surface can be derived through precise linear regression $\left(\mathrm{R}^{2}(1)\right)$. Fractal dimension corresponding to reject surface is 2.47 , while that of fine quality surface reveals 2.38. In addition, the regression values corresponding to the method of morphological envelopes is also included $\left(\mathrm{R}^{2}(2)\right)$.

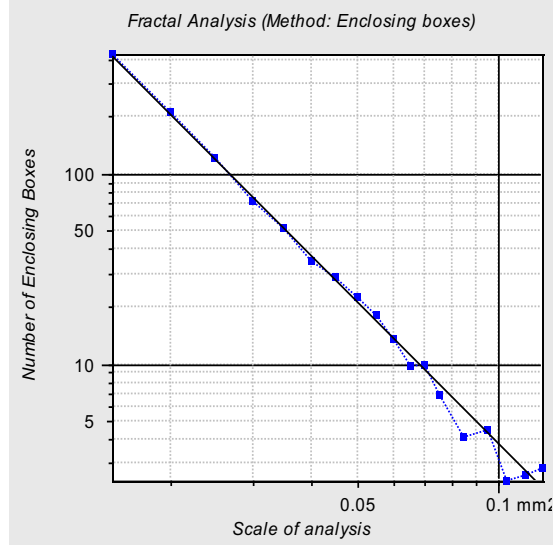

Fractal dimension: 2.47

$R^{2}(1): 0.996$ $R^{2}(2): 0.989$

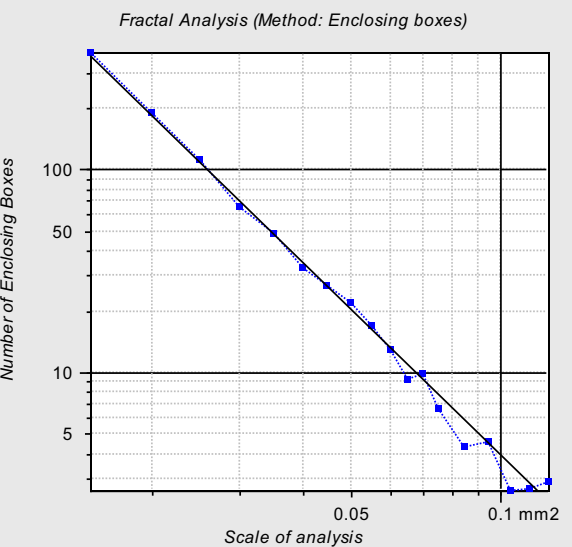

Fractal dimension: 2.38

$R^{2}(1): 0.997$

$R^{2}(2): 0.99$

FIGURE 3. Analysis of fractal dimensions of reject (left) and fine (right) surfaces.

Finally, it might be noted that the contactless scanning of surface structures provides useful approach to compare tendency of various highly filled systems to flow-induced separation occurring during their processing. Such analysis connected to flow-induced separation of the molded parts obtained from the special designed testing mold quantified via SEM/EDX and rheological properties during molding should lead to successful simulation, and consequently reduction/elimination of the issue.

\section{ACKNOWLEDGMENTS}

The author (B.H.) would like to acknowledge the Grant Agency of the Czech Republic (Project No. 103/08/1307). This article was written with support of Operational Program Research and Development for Innovations co-funded by the ERDF and national budget of Czech Republic, within the framework of project Centre of Polymer Systems (reg. number: CZ.1.05/2.1.00/03.0111).

\section{REFERENCES}

1. M. Thornagel, "MIM-simulation: A virtual study on phase separation" in Euro PM2009, Proceedings of EURO PM, European Powder Metallurgy Association, Shrewsbury, UK, 2009, pp. 135-140.

2. L. Jiranek, B. Hausnerova, T. Hartwig, Community Design 001704974, Office for Harmonization in the Internal Market, Alicante (6 May, 2010)

3. B. Hausnerova, L. Marcanikova, P. Fillip and P. Saha, "Wall-Slip Velocity as a Quantitative Measure of Powder-Binder Separation during Powder Injection Moulding", in World PM2010, Proceedings of World PM2010 Congress, European Powder Metallurgy Association, Shrewsbury, UK, 2010, p. 557-562.

4. M. Jenni, L. Schimmer, R. Zauner, J. Stampfl, J. Morris, PIM International 2, $50-55$ (2008). 
Copyright of AIP Conference Proceedings is the property of American Institute of Physics and its content may not be copied or emailed to multiple sites or posted to a listserv without the copyright holder's express written permission. However, users may print, download, or email articles for individual use. 